East African Medical Journal Vol. 86 No. 1 January 2009

MANAGEMENT OF BILATERAL TEMPOROMANDIBULAR JOINT ANKYLOSIS IN CHILDREN: CASE REPORT

M. K. Akama, BDS, MDS, Lecturer, Department of Oral and Maxillofacial Surgery, Oral Medicine and Oral Pathology, S. Guthua, Professor, BDS, MMedSc, COMS, FIAOMS, FCS, M. L. Chindia, BDS, MSc, FFDRCSI, Associate Professor, Department of Oral and Maxillofacial Surgery, Oral Medicine and Oral Pathology, and S. K. Kahuho, MBChB, FFARCS (Ire), DA (Lond), Division of Anaesthesia, Department of Surgery, College of Health Sciences, University of Nairobi, P. O. Box 19676-00202, Nairobi, Kenya

Request for reprints to: Dr. M. K. Akama, Department of Oral and Maxillofacial Surgery, Oral Medicine and Oral Pathology, College of Health Sciences, University of Nairobi, P. O. Box 19676-00202 Nairobi, Kenya

\title{
MANAGEMENT OF BILATERAL TEMPOROMANDIBULAR JOINT ANKYLOSIS IN CHILDREN: CASE REPORT
}

\author{
M. K. AKAMA, S. GUTHUA, M. L. CHINDIA and S. K. KAHUHO
}

\begin{abstract}
SUMMARY
Long standing temporomandibular joint (TMJ) ankylosis may cause severe facial deformity and growth retardation as well as psychological problems when it occurs in children. Different kinds of opinion have been advanced as regards its management. This article discusses the aetiology and management of bilateral TMJ ankylosis in form of a case report in a patient with severe facial deformity, malocclusion and retarded growth following bilateral TMJ ankylosis secondary to trauma. The patient was successfully managed by placement of costochondral grafts and interpositional arthroplasty with partial temporal myofascial flaps. Good mouth opening was achieved and subsequent follow-ups showed improved mandibular and general body growth as well as psychosocial performance. The aim of this paper is to discuss the management challenges posed by bilateral TMJ ankylosis.
\end{abstract}

\section{INTRODUCTION}

The word ankylosis is derived from a Greek terminology meaning 'stiff joint'. Ankylosis of the TMJ may either be true (intra-articular) or false (extraarticular), bony or fibrous. The commonest causes of ankylosis are trauma and infection in or around the joint region. Other causes that have been cited include tumours and inflammation. Children have a greater tendency towards post-traumatic ankylosis, mostly before 10 years of age (1). The factors that predispose to ankylosis include meniscal tear, age (10 years and below) and prolonged immobilisation in patients with intracapsular injuries (2). When it occurs in a child, not only does it have serious effects on the future growth of the jaws and teeth, but also profound influence on the psychosocial development of the patient because of the obvious facial deformity which worsens with growth. It is crucial that early diagnosis and management are undertaken if the worst sequelae of this condition are to be avoided.

\section{CASE REPORT}

A nine year old boy from Somalia was referred to the oral and maxillofacial surgery clinic of the University of Nairobi Dental Hospital for management of bilateral TMJ bony ankylosis. The ankylosis developed following bilateral condylar fractures as result of falling from a height at the age of five years. The boy hardly received any care in Somalia due to lack of maxillofacial services. At presentation, he appeared small for his age, otherwise his general condition was good. On clinical examination, therapeutic scars inflicted by traditional healers were noticed on the parasymphyseal region bilaterally and the circumoral area. Mandibular retrognathia was apparent on the side profile. The dentition was in the mid mixed stage. An orthopantomogram revealed moderate antigonial notching. There was limited mouth opening with no palpable movement over both joints. Affordable radiographs included orthopantomogram and bilateral TMJ tomograms (Figure 1). 
Figure 1

OPG showing bilateral TMJ ankylosis

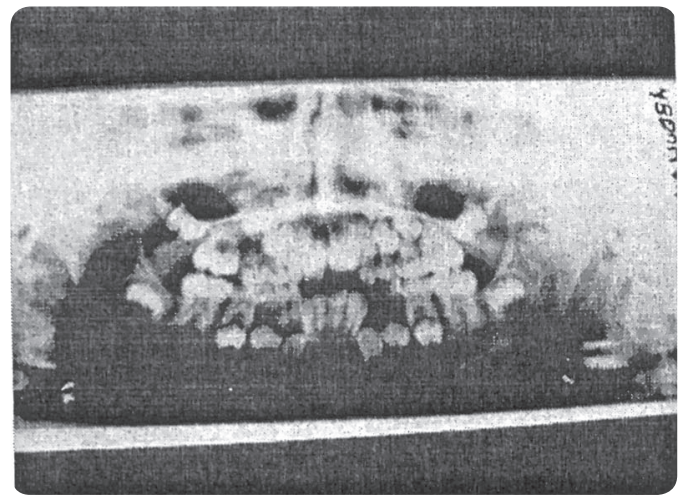

These images showed mushroom shaped radioopacities confirming bilateral TMJ ankylosis with birateral hyperplasia of the coronoid processes. A treatment plan was designed which included the following:

(i) Primary major surgical procedures

Bilateral release of the bony ankylosis through a preauricular approach.

Bilateral coronoidectomies.

Reconstruction of theTMJswithcostochondral grafts (CCG) with internal semi-rigid fixation and interposition partial temporal myofascial flaps.

(ii) Physiotherapy

Aggressive mouth opening exercises.

(iii) Orthodontic treatment.

(iv) Secondary surgical procedures.

Orthognathic surgery.

The surgery was planned to be done under general anaesthesia. Anaesthetic difficulties were anticipated as the mouth opening was nil and fibreoptic facility was not available. It was thought pragmatic to attempt blind nasal intubation first, failure to which tracheostomy was to be done. Anaesthesia was induced and blind nasotracheal intubation was attempted. Adequate mask ventilation, oxygen saturation and anaesthetic depth was ensured before introducing a lubricated nasotracheal tube. After three unsuccessful attempts at entering the trachea, nasopharyngeal insuflation was done by placing the tube in the nasopharyngeal region. Through preauricular approach (Figure 2), both ankylosis were released and adequate mouth opening to allow rigid laryngoscopy was achieved. The endotracheal tube was then introduced into the trachea. The tube position was confirmed by chest auscultation. It was then secured, and general anaesthesia continued accordingly.

Bilateral resection of the ankylotic mass (Figure3) was completed as well as bilateral coronoidectomies through the same incision. Maximum mid intercisial distance of $40 \mathrm{~mm}$ was achieved. The temporal bone on both sides was recontoured with a round bar to create a glenoid fossa. Harvesting of the costochondral grafts was done, the donor site being the right fifth and sixth ribs.

Figure 2

Extension of pre-auricular incision

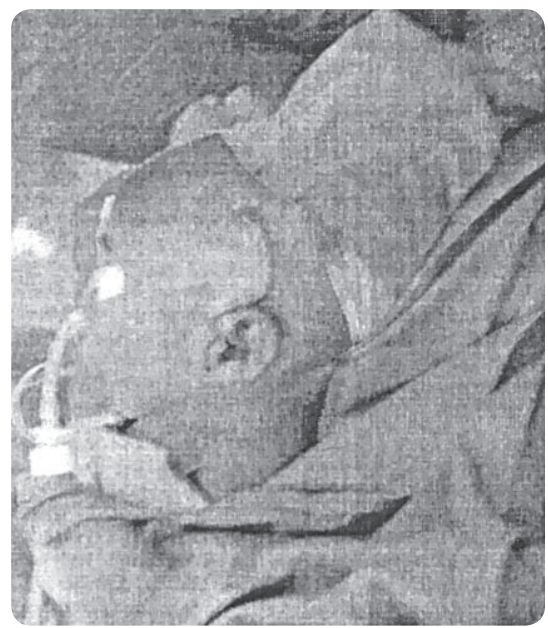

Figure 3

Total bony ankylosis of the TMJ

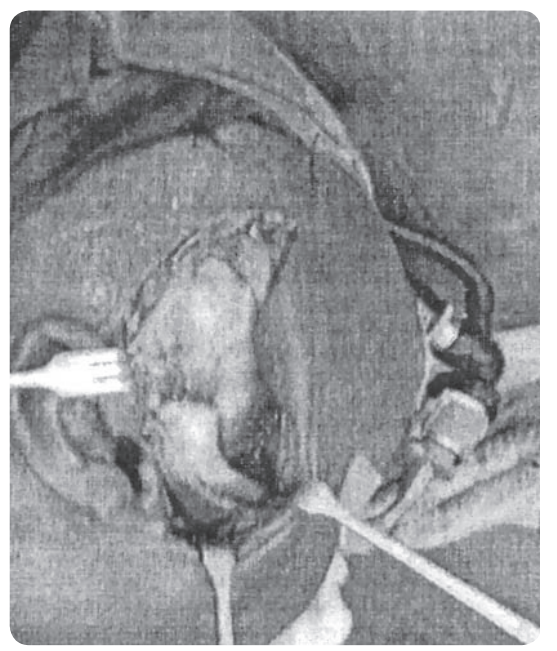

This was achieved by making a $5 \mathrm{~cm}$ infra mammary incision over the costochondral junction of the $5^{\text {th }}$ rib. The periosteum was carefully stripped off the rib starting distally and advancing proximally towards the perichondrium. Care was taken not to separate the cartilaginous part of the graft from bone by leaving a strip of the outer perichondrium to join the two. The parietal perichondrium was stripped off the graft to avoid perforating the pleura. The bony part of the graft was resected with rib cutters whereas the chondral part was resected with a blade. Dissection was done inferiorly to expose the $6^{\text {th }}$ rib and a similar procedure was followed to harvest the graft. After ascertaining that there was no perforation of the parietal pleuera, the periosteum for each rib was approximated and wound closed in layers. The CCGs (Figure 4) were 
contoured to achieve proper articulation with the glenoid fossae on the temporal bone. To allow good access to the ramus, an additional submandibular incision was made bilaterally whereas to improve on semi-rigid fixation of the graft, superficial vertical dicortication was done on both rami to accommodate at least two-thirds of the CCGs. This ensured good initial stability and integration of the graft with the mandible.

Figure 4

Contoured costochondral end of the rib

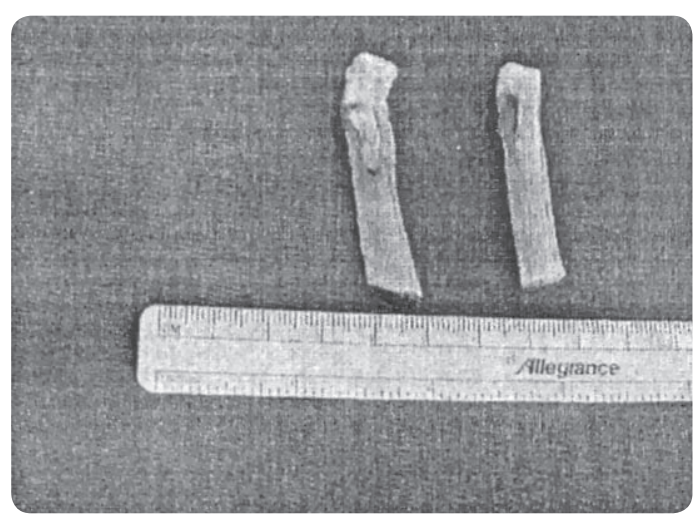

A partial temporal myofascial flap was raised and rotated bilaterally to cushion and act as the TMJ meniscus. It was rotated and interposed between the graft and the temporal bone.

The graft was then fitted in the groove on the ramus on each side and secured with two $0.5 \mathrm{~mm}$ stainless steel wires. Vacuum drains were inserted followed by closure of wound in layers and crepe bandage applied. Physiotherapy was commenced on the second postoperative day. Weakness of both frontal branches of the facial nerve was noted during the first post-operative day. This had not resolved at the time of discharge seven days post operatively, however, some improvement was noted two weeks later and complete recovery in one month. Mouth opening was good with an inter-incisal distance of $36 \mathrm{~mm}$.

\section{DISCUSSION}

The causes of TMJ ankylosis that have been documented includeinfection, trauma, inflammatory diseases and neoplasms. However, trauma and infection have been identified as the two leading causes. Laskin (3) evaluated the factors which could contribute to the development of post-traumatic TMJ ankylosis and summarised them into the age of the patient, site and type of fracture, duration of immobilisation and damage to the meniscus. If the cause of TMJ ankylosis is trauma as was in this case and a case published earlier (4), it is thought that the state of the meniscus may be the key factor in determining whether or not post-traumatic ankylosis will develop. Perforation of the meniscus predisposes to ankylosis. TMJ ankylosis in children leads to impaired mandibular growth resulting in mandibular retrognathism and attendant functional and cosmetic defects as well as feeding difficulties and poor oral hygiene. There is potential danger of airway embarrassment in the event of life-threatening odontogenicinfections, for example Ludwigs Angina. Therefore, treatment should be initiated as soon as the condition is recognised, with the main objective of re-establishing joint function and harmonious jaw movements (5).

TMJ ankylosis presents a serious anaesthetic challenge as rigid laryngoscopy is not possible. The report of the confidential enquiry into peri-operative deaths for England and Wales, 1986, revealed that difficult or failed intubations contributed to six of the 4034 deaths reported (6). Administration of anaesthesia to patients with TMJ ankylosis ideally requires the availability of flexible fibreoptic bronchoscope, trained personnel who use the equipment and monitoring facilities. These facilities however, are lacking in many developing countries. Blind intubation has its disadvantages which include high failure rate even in the hands of an expert, bleeding due to local trauma, secretions in the airway and distorted tissue anatomy which makes it even more difficult especially in children(7) Naveed et al (8) have described a technique of facilitating intubation by prior placement of an endotracheal tube into the oesophaghus followed by blind insertion of another endotracheal tube into the trachea. These techniques of blind intubation may be helpful in reducing the psychological trauma and complications of elective tracheostomy.

There are three basic surgical objectives in the treatment of TMJ ankylosis. These are to establishjoint movement, to prevent relapse and to achieve normal growth and development. Various autogenous grafts have been used in the reconstruction of the TMJ. These include the fibula, clavicle, sternoclavicular joint, iliac bone, metatarsal bone and metatarsal-phalangeal joint. The costochondral graft has however gained popularity over the others in recent times (5). It offers several advantages which include biological and anatomic similarity to the mandibular condyle, low morbidity of the donor site, ease in obtaining and adapting the graft and the regenerative potential in the growing child $(9,10)$. It has almost similar primary and secondary cartilage to those of the mandibular condyle and therefore it is thought that its growth potential is similar to that of the condyle. It acts as an adaptive centre to promote symmetrical growth of the mandible and function as well as correction of mandibular retrognathia and laterognathia which overall improves the psychologic outlook for both the patient and parents. Other operative techniques have been described which include gap arthroplasty, 
interpositional arthroplasty with lyophilized cartilage and reconstruction of thejoint with alloplastic material (5). In our centre, we routinely use costochondral graft in TMJ reconstruction in children. Most of these techniques have unsatisfactory results, for example, the growth of the condyle is not fully restored and a procedure like gap arthroplasty often results in anterior open bite and re-ankylosis in addition to diminished mandular growth (5).

One of the goals of the treatment of ankylosis is to prevent re-ankylosis. Topazian's (11) review of gap arthroplasty without interposition reported a recurrence rate as high as 53\%.To reduce the chance of re-ankylosis, some authors suggest radical condyle and neck removal as well as coronoidectomy (12). This has got the disadvantage of greatly reducing the vertical ramus height. Re-ankylosis is less likely if a material is interposed between the divided bone ends (13). Autogenic tissues have gained popularity over allogenic materials because the latter tend to initiate foreign body reaction (2). Interposition arthroplasty using locally available vascularised temporalis myofascial flap has been found to be a reliablemethod to prevent re-ankylosis (2). It obviates the disadvantages of alloplastic materials as well as non-vascularised autogenous tissues.

A protocol for TMJ management has been developed (5). This includes:

(i) Aggressive resection or the ankylotic mass.

(ii) Bilateral coronoidectomy.

(iii) Lining or the fossa with temporalis muscle and fascia.

(iv) Reconstruction of the condyle with a costochondral graft.

(v) Rigid or semi-rigid fixation of the graft.

(vi) Early mobilisation and aggressive physiotherapy.

The current case was managed along these lines. The patient remains under close follow-up. He may require orthognathic surgery later but for now he has been referred for routine dental care and orthodontic evaluation and management.

\section{ACKNOWLEDGEMENTS}

To the management of the University of NairobiDental Hospital for allowing us to publish this work.

\section{REFERENCES}

1. Williams, J.L.I. Injuries of the condylar and coronoid process: In: Rowe and Williams Maxillofacial Injuries $2^{\text {nd }}$ ed. Churchill Livingstone. 1994; pp417.

2. Ahmaed, Q.G., Siddiqui, R.A., Khan, A.H. andSharma S.C. Interposition arthroplasty in temporomandibular joint ankylosis. Indian J. Otolanyngology and Head and Neck Surg. 2004; 56: 1-8.

3. Laskin, D.M. Role of the meniscus in the aetiology of post-traumatic temporomandibular joint ankylosis. Intern. J. Oral Surg. 1978; 7: 340.

4. Guthua, S.W., Maina, D.M. and Kahugu, M. Management of post traumatic temporomandibular joint ankylosis in children: Case report. East Afr. Med. J. 1995; 72: 471-475.

5. Kaban, L. B., Perott, D.H. and Fisher K.A. Protocal for management of TMJ ankylosis. J. oral Maxillofacial Surg. 1990;48: 1145-1151.

6. American society of Anaesthesiologists, Committee on professional liability. Preliminary study of closed claims. ASA Newsletter. 1988; 52: 8-10.

7. Thompson, A.E. Issues in airway management in infants and children. Respir. Care. 1999; 44: 650.

8. Naveed, M. and Sayeed, A. Facilitated blind nasotracheal intubation in paralysed patients with temporomandibular joint ankylosis. JCPSP. 2005: 15: 4-6.

9. Guyuron, B. and Lasa, C.I.Jr. Unpredicatable growth pattern of costochondral graft. Plast. Reconstr. Surg. 1992; 90:880-886.

10. Figueroa, A.A., Gans B. J. and Prunzansky, S. Longterm follow-up of mandibular costochondral graft. Oral. Surg. Oral. Med. Oral. Pathol. 1984; 58: 257-268.

11. Topazian, R.G. Etiology of ankylosis of the temporomandibular joint analysis of 44 cases. J. Oral Surg. 1964; 22: 227.

12. Rajgopal, A., Banerjee, P.K., Baluria V. and Sural A. Temporomandibular joint ankylosis: A report of 15 cases. J. Maxillofacial Surg. 1983; 11:37.

13. Barry, M. The temporomandibularjoint. In: Mc Carthy (Ed) Plastic surgery: W.B. Saunder's Company. 1990; 1475-1513. 